\title{
The self gravity effect on the orbital stability of Twotinos
}

\author{
YUSUKE TSUKAMOTO \\ ${ }^{a}$ Department of Astronomy, University of Tokyo,7-3-1 Hongo, Bunkyo-ku, Tokyo 113-0033, Japan; tukamoto@cfca.jp
}

\begin{abstract}
We investigate how the self gravity of Twotinos changes its orbital stability using N-body simulations in which the gravitational interaction between planetesimals are fully calculated. We show the timescale in which the
\end{abstract} half of Twotinos becomes unstable, $t_{\text {half }}$, obey the formula,

$$
t_{\text {half }}=4 \times 10^{6}\left(\frac{M_{\text {tot }}}{0.1 M_{\oplus}}\right)^{-1}\left(\frac{m_{p}}{7.6 \times 10^{23} g}\right)^{-1}\left(\frac{\left\langle i^{2}\right\rangle^{1 / 2}}{0.002}\right)(\text { years }),
$$

, if we assume the primordial planetesimals disk have the power low surface mass density, $\Sigma=\Sigma_{0} \times r^{-2 / 3}$. Where $M_{t o t}, m_{p},\left\langle i^{2}\right\rangle^{1 / 2}$ are the total mass of large bodies of Twotinos, the maximum mass of planetesimals, and the inclination dispersion. With this formulae, we conclude the total mass of Twotinos is reduced to the order of $0.01 M_{\oplus}$ by the self gravity and secular perturbation of Planets even if there was huge mass such as several order of the earth mass in 1:2 MMR of Neptune at the early age of the solar system. These results will invoke reexamination to many previous works explaining the dynamical evolution of TNOs.

Keywords: TNOs, KBOs, planetary system, Twotinos, mean motion resonance, the Solar System, $n$-body, GRAPE-6

\section{Introduction}

It is important feature that the highly eccentric planetesimals in the mean motion resonance (MMR) of Neptune such as Plutinos or Twotinos exist and stay in for the age of the solar system. These populations have provided the informations to establish the dynamical evolution model of the primordial Solar system. Malhotra (1995) proposed Resonant sweeping model in which Neptune migrate several AU capturing primordial TNOs into its MMR. This model firstly explain the Pluto's peculiar orbit and successfully predict the existence of Plutinos, Twotinos, and scattered TNOs. But many unexplainable orbital characteristics of TNOs remain. After Malhotra's work, lots of works have been done to explain the orbital structures of TNOs more precisely. These works are categorized into two types.

The works categorized into first type follow the framework of Malhotra's work and carefully investigate how the resulting structure of TNOs is changed by the initial position, migration model of planets or the initial distribution of planetesimals disk. For example, by changing the migration speed of Neptune, Ida et al. (2000) try to explain the ratio of Plutinos to Twotinos. Zhou et al. (2002) also address this issue considering the Preprint submitted to Elsevier 
stochastic migration model of Neptune. Levison \& Morbidelli (2003) propose a bolder model in which the primordial planetesimal disk was assumed to be dynamically cold and truncated around 35AU. If the mass of Twotinos was about $3 M_{\oplus}$ when the 1:2 MMR of Neptune reached 35AU and the migration of Neptune after that was properly stochastic, the outcome can accounts for almost all aspects of TNOs region. This model is very attractive because of its strong explanation for the structure of TNOs. But the strong stochastic motion of Neptune by planetesimals is unlikely because the mass of planetesimals are too small to induce strong stochastic migration (Murray-Clay \& Chiang 2006).

Nice model (Tsiganis et al. 2005, Gomes et al. 2005, Morbidelli et al. 2005) seems to be the most prosperous model to explain the dynamical structure of TNOs. In this model, the planetesimal disk is also assumed to be truncated around $35 \mathrm{AU}$ and four giant planets was formed in more compact configuration than current configuration. During the early migration phase, Jupiter and Saturn was locked into the mutual 1:2 MMR and this resonance induced strong instability to Uranus and Neptune. By this instability, They were scattered outwards shaking up the outer planetesimal disk and reduce their eccentricity by the dynamical frictions from planetesimals. This model accounts for not only all important aspects of TNOs region but also the Late Heavy Bombardment, the Trojan populations of Jupiter, the eccentricity and inclination of the planets. Nice model is very powerful but it assumes a lot of the conditions for the primordial solar system and whether such a dynamical migration really occur or not is still uncertain. Recently, Minton \& Malhotra (2009) suggested that the information of the migration process of planets was graved into the asteroid belt. Thus, what kind of migration occurred in early solar system will revealed by the detailed investigations of the asteroid belt.

The works belong to the second type consider the orbital instability after the migration. The instability of the orbit may change the structure of TNOs. Many authors (Levison and Stern 1995, Malhotra 1996, Morbidelli 1997, Nesvorný and Roig 2000, 2001, Tiscareno \& Malhotra 2009) have investigate the stability of the orbits in the resonant region under the perturbation from the Planets. Levison \& Stern (1995) studied the stability of the orbits in the 2:3 MMR as a function of $A_{\sigma}, A_{\omega}$ where $A_{\sigma}, A_{\omega}$ are the initial amplitudes of the resonant angle and the argument of perihelion. They found that $A_{\sigma}>120^{\circ}$ were unstable over $4 \times 10^{9}$ years even if $A_{\omega}=0$ and as $A_{\omega}$ increases, the boundary value of $A_{\sigma}$ which separates stable orbits and unstable orbits decreases. As they have shown, the amplitude of resonant angle determine the stability of orbits.

Nesvorný \& Roig (2001) studied the orbital stability of 1:2 MMR using Lyapunov characteristic exponent. Their result showed that the orbits which have $A_{\sigma}>30^{\circ}, e=0.3 i=5^{\circ}$ are unstable for the age of the Solar System. On the other hand, they pointed out to determine the stable region of intermediate eccentricity such as $0.1<e<0.4$ is very difficult because of its complex chaotic nature. But figure 5 in their paper seems to show $A_{\sigma}>30^{\circ}$ can be a crude threshold between stable and unstable orbit for $0.1<e<0.4$ and we use this value as the threshold in this paper. They also pointed out the additional kick to the amplitude of the resonant angle may play an important role on the orbital stability of the Twotinos (see, fig 10 of the Nesvorný \& Roig 
2001).

Although the gravitational effect of planets on the orbital evolution of TNOs are well investigated, the study about the mutual gravity effect between planetesimals is very rare. The gravitational effect on Plutinos by the back ground planetesimals is partially studied in Levison and Stern (1995) by the particle-in-box framework. In their work, the gravitational effect to Pluto from background particles which have circular orbits and the size between $1 \mathrm{~km}-330 \mathrm{~km}$ is considered. They conclude that the orbit of Pluto can be stabilized by the effect. However, Their investigation is limited and it is important to investigate the mutual gravitational effect of resonant TNOs more precisely.

In this paper, we address the issue how the self gravity in the resonant TNOs affects its stability of the planetesimals with N-body simulation in which the self gravity is fully considered. Especially, we investigate Twotinos because the stable region in the phase space of Twotinos is smaller than that of Plutinos and Twotinos seem to be destabilized easier than Plutinos and they may affect the structure of TNOs more strongly. The analysis about 2:3 MMR will be done in future works.

The numerical method and the initial conditions are described in Section 2, In Section 3, we show our results and estimate the effects of the self gravity on the actual Twotinos by extrapolating the results. We summarize the results in Section 4

\section{Method of Calculation and Initial Conditions}

\subsection{Integration Method}

We use the fourth-order Hermite scheme (Makino \& Aarseth 1992) with hierarchical time-steps (Makino 1991) improved for planetary systems (Kokubo et al. 1998 ) for numerical integration of the planetesimals and Neptune. The gravitational interaction between planetesimals are fully considered and the gravitational effect of planetesimals to Neptune or the Sun are neglected. We use position of the Sun as a origin of the coordinate for planetesimals and Neptune. We use the equation of motion for planetesimals as follow

$$
\begin{aligned}
\mathbf{a}_{i} & =-\sum_{i \neq j} G m_{j} \frac{\mathbf{r}_{\mathbf{i j}}}{r_{i j}^{3}}-G\left(m_{i}+M_{\odot}\right) \frac{\mathbf{r}_{\mathbf{i}}}{r_{i}^{3}} \\
& -G M_{N}\left(\frac{\mathbf{r}_{\mathbf{i N}}}{r_{i N}^{3}}+\frac{\mathbf{r}_{\mathbf{N}}}{r_{N}^{3}}\right)
\end{aligned}
$$

where $M_{\odot}, M_{N}, r_{i}$, and $r_{i N}$ are the mass of the Sun, the mass of Neptune, position of planetesimal relative to the Sun and that relative to Neptune. $r_{N}$ is position of Neptune relative to the Sun. The gravitational forces of planetesimals to Neptune is neglected i.e., the equation of motion for Neptune is that of simple two body problem.

We use GRAPE-6 (Makino et al. 2003) and GRAPE-6A (Fukushige et al. 2005) to calculate the gravitational interaction between planetesimals. For both the planetesimals and Neptune, we use the standard 
time-step criterion (Aarseth 1985)

$$
\Delta t=\sqrt{\eta \frac{|a|\left|a^{(2)}\right|+|\dot{a}|^{2}}{|\dot{a}|\left|a^{(3)}\right|+\left|a^{(2)}\right|^{2}}} .
$$

Where $a, \dot{a}, a^{(n)}$ are the acceleration and its first order, n-th order time differential.

The $\eta$ is selected as the energy error of planetesimals is less than $0.003 \%$ of initial energy of planetesimals. Each orbit is integrated with several hundred time-steps on average. This is sufficient to avoid the artificial effect on the orbit such as precession.

\subsection{Initial Conditions}

We make the initial resonant population by simple resonant capture process by Neptune. At first, the planetesimals are distributed as a cold disk whose distributions of eccentricity and inclination are both given by Rayleigh distribution with dispersion $\left\langle e^{2}\right\rangle^{1 / 2}=2\left\langle i^{2}\right\rangle^{1 / 2}=0.004$ and its mass is equal to zero. The number density of the disk is power law of the distance from the Sun and its exponent is equal to $-3 / 2$. The inner and outer cutoff of the disk are $25 \mathrm{AU}$ and $50 \mathrm{AU}$, respectively. We use 10000 particles to make sufficient number of resonant particles. After migration of Neptune, we picked up the resonant particles for initial conditions. In our model, only Neptune and Sun are considered and the gravitational effects of other Planets are neglected to investigate the mutual gravitational effect of TNOs genuinely.

The migration model is similar to that of Malhotra (1995) and the semi-major axis of Neptune evolve such as

$$
a(t)=a_{f}-\left(a_{f}-a_{i}\right) e^{-t / \tau}
$$

where $\tau$ is migration timescale and its value is $5.0 \times 10^{6}$ years. $a_{f}, a_{i}$ are the final position and initial position of Neptune and we fix these parameters. the value of the parameters are $23 \mathrm{AU}$ and 30 AU, respectively. As Gomes et al (2004) investigated, the migration mode of Neptune drastically change due to the mass and the surface mass density profile of the planetesimal disk and there are many possibilities for the migration model. However, we adopt the typical migration model to make our initial conditions for simplicity. The system is integrated for $1.6 \times 10^{7}$ and Neptune migrate from 23 AU to 29.7 AU trapping the planetesimals into its resonance.

The figure 1 shows our initial disk and the result after Neptune migration. Using cold disk, the resonant capture is very effective and all particles swept by the 1:2 and 2:3 MMR of Neptune are captured into the resonance. The perturbation from other planets reduce the capture probability and this is some ideal results. But this condition is good for investigate the genuine effect of the self gravity. From now on, we focus on the planetesimals which captured into the 1:2 MMR.

We pick up 1000 (model 1, 6, 7, 8), 2000 (model 2,4), 500 (model 3, 5) particles from the 1:2 MMR 
population and assign mass. We define the planetesimals which captured in the 1:2 MMR as those whose semi-major axis is $46.7 A U<a<47.7 A U$.

In the model 1 , The total mass of Twotinos is about $0.1 M_{\oplus}$ this correspond to the surface mass density which is about 100 times smaller than that of the minimum-mass solar nebular model (Hayashi et al 1981). The diameter of each particle is about $450 \mathrm{~km}$. The detailed parameters are shown in Table 1. In model 2-5, we change the total mass and the mass of planetesimals to see the dependence of the relaxation time on these parameters. In model 6,7 , we change the inclination dispersion to see the effect of inclination. We make these models by changing the dispersion of inclination when the Neptune migration is over. The model 8 is the model of massless case and it is used for comparison with other models. We integrate the system for $1.6 \times 10^{7}$ years considering the gravitational interaction between the planetesimals. The gravity to Neptune from planetesimals are neglected to see the genuine internal gravitational effect.

The figure 2 shows the initial distribution of planetesimals on $a-e$ plane and $e \cos \left(\sigma_{1: 2}\right)-e \sin \left(\sigma_{1: 2}\right)$ plane, where $\sigma_{1: 2}$ is the resonant angle, $\sigma_{1: 2}=2 \lambda-\lambda_{N}-\varpi$. Most particles are trapped around $\left\langle\sigma_{1: 2}\right\rangle \simeq 270^{\circ}$. This asymmetric capture is agree well with the result of Chiang \& Jordan (2002).

Table 1: The initial conditions

\begin{tabular}{cccccc}
\hline \hline Model & $N$ & $M(\mathrm{~g})$ & $r(\mathrm{~km})$ & total mass $\left(M_{\oplus}\right)$ & $\left\langle i^{2}\right\rangle^{1 / 2}$ \\
\hline 1 & 1000 & $7.62 \times 10^{23}$ & 450 & 0.127 & 0.002 \\
2 & 2000 & $3.81 \times 10^{23}$ & 360 & 0.127 & 0.002 \\
3 & 500 & $1.52 \times 10^{24}$ & 570 & 0.127 & 0.002 \\
4 & 2000 & $7.62 \times 10^{23}$ & 450 & 0.254 & 0.002 \\
5 & 500 & $7.62 \times 10^{23}$ & 450 & 0.0635 & 0.002 \\
6 & 1000 & $7.62 \times 10^{23}$ & 450 & 0.127 & 0.01 \\
7 & 1000 & $7.62 \times 10^{23}$ & 450 & 0.127 & 0.02 \\
8 & 1000 & 0 & 0 & 0 & 0.002 \\
\hline
\end{tabular}

\section{Results}

Figure 3 shows the snapshot of model 1 and model 8 (massless case) at $t=1.6 \times 10^{7}$ years. The top right panel shows the snapshot on $e \cos \left(\sigma_{1: 2}\right)-e \sin \left(\sigma_{1: 2}\right)$ plane of the model 1. The particles distribute broad region. This means the libration of resonant angle are amplified by the self gravity. Because the amplitude of resonant angle correlate with the semi-major axis, the amplitude of semi-major axis is also amplified as shown in the top left panel of the figure. Compared with the figure 5 of Nesvorný and Roig (2001) and the top left panel of the figure, We can see that many planetesimals become unstable. On the other hand, we can not find any obvious change in the result of model 8 (bottom panels) over the integration time.

We show the plot of the planetesimals on $\left\langle\sigma_{1: 2}\right\rangle-A_{\sigma_{1: 2}}$ plane of the resonant angle in figure 4 We take snapshot each 1,600 year and the mean value, $\left\langle\sigma_{1: 2}\right\rangle$ is averaged value of the resonant angle over 160,000 year. 

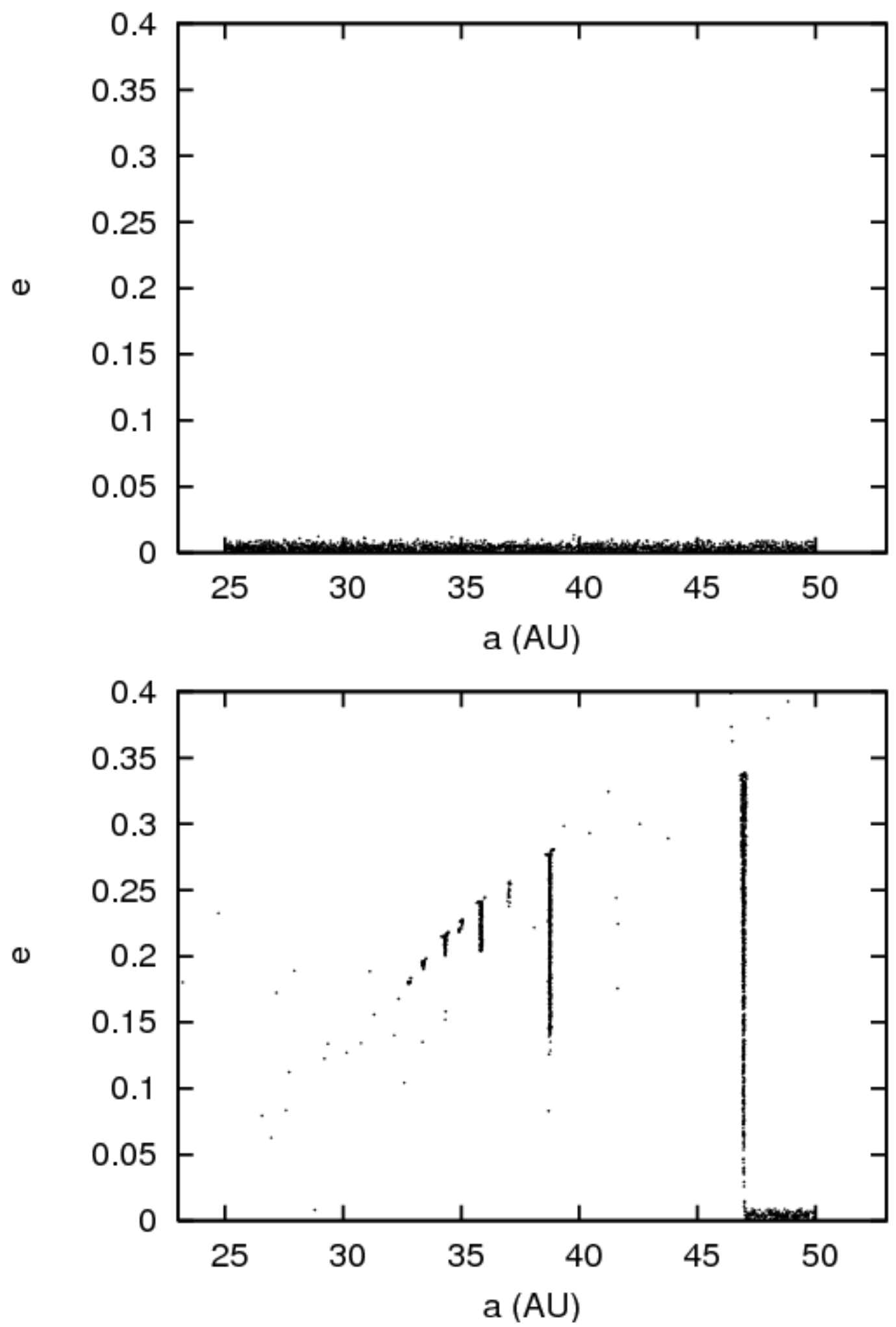

Figure 1: The a-e plot of the initial planetesimals disk (top) and the disk after sweeping by MMR of Neptune (bottom). The resonant capture process is effective and the $100 \%$ of the particles which encounter the 2:3 or 1:2 MMR are captured into the resonances. 

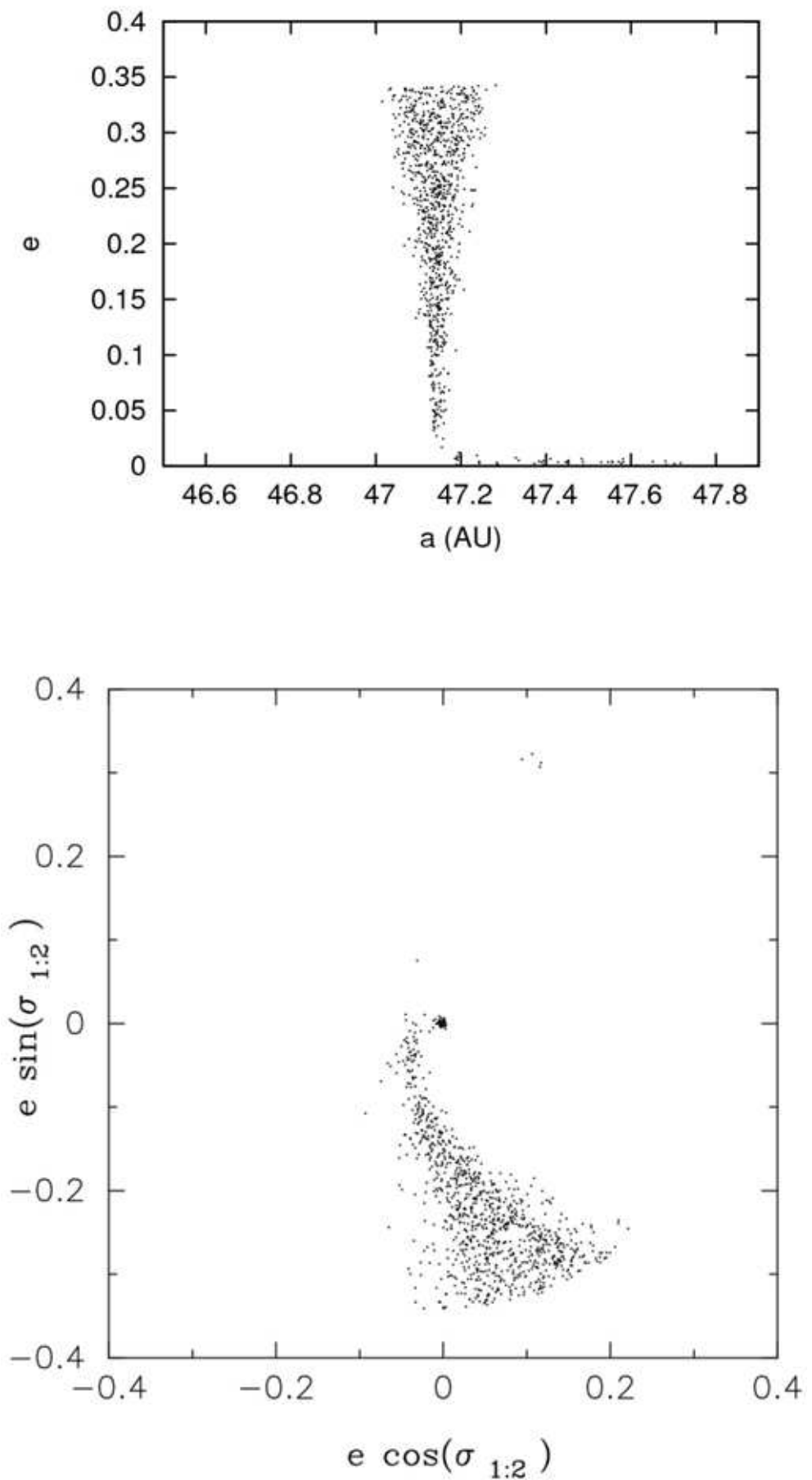

Figure 2: The a-e plot (top) and $e \cos \left(\sigma_{1: 2}\right)-e \sin \left(\sigma_{1: 2}\right)$ plot (bottom) of the initial condition for our simulations, where $\sigma_{1: 2}$ is the resonant angle $\sigma_{1: 2}=2 \lambda-\lambda_{N}-\varpi$. 
The amplitude, $A_{\sigma_{1: 2}}$ is defined as $\left(\sigma_{\max }-\sigma_{\min }\right) / 2$. The $\sigma_{\max }$ and $\sigma_{\min }$ are maximum and minimum value of resonant angle each 160,000 year. The "window time", 160,000 year is 1-10 times longer than the period of oscillation of $\sigma_{1: 2}$ and it is enough to estimate the mean, maximum, minimum values. The bottom panel shows the result of model 1 and we can see the three populations. The two populations whose amplitudes are less than $80 \mathrm{deg}$ are correspond to the populations whose orbits are asymmetric and tadpole like on $e \cos \left(\sigma_{1: 2}\right)$ $e \sin \left(\sigma_{1: 2}\right)$ plane and the population whose $\left\langle\sigma_{1: 2}\right\rangle$ is around $180^{\circ}$ is the population whose orbits is symmetric and horse-shoe like orbit. The existence of these three population is consistent with the result of Chian and Jordan (2002) (see, figure 7 of their paper). The result of massless case (top panel) does not show any obvious change.

Note that The planetesimals distribution which have $\left\langle\sigma_{1: 2}\right\rangle \simeq 180^{\circ}$ and $A_{\sigma_{1: 2}} \simeq 180^{\circ}$ on top panel is those which is ejected from 1:2 MMR. The planetesimals distribution which have $220^{\circ}<\left\langle\sigma_{1: 2}\right\rangle<250^{\circ}$ and $A_{\sigma_{1: 2}} \simeq 20^{\circ}$ on bottom panel is those which have low eccentricity $(e<0.1)$. For those planetesimals, the two islans on $e \cos \left(\sigma_{1: 2}\right)-e \sin \left(\sigma_{1: 2}\right)$ plane are around $\sigma_{1: 2} \simeq 250^{\circ}, 70^{\circ}$ (see, top right panel of figure 6 of Murray-Clay \& Chiang (2005)).

We show an example of the time evolution of the eccentricity and the resonant angle of a planetesimal with (red line, model 1) and without (green line, model 8) self gravity in figure 5 . The random walk nature is seen in the change of the eccentricity of red line. The top right panel shows the time evolution of the resonant angle. Around $4 \times 10^{6}$ year, the amplitude of resonant angle changes discontinuously for the red line. This shows the orbit of the particle on the $e \cos \left(\sigma_{1: 2}\right)-e \sin \left(\sigma_{1: 2}\right)$ plane transit from tadpole orbit to horse-shoe orbit. The transit from tadpole orbit to horse-shoe orbit and vice-vase occur several time. This shows that the particles can easily across the separatrix due to self gravity. This nature is also displayed in the Chiang and Jordan (2002). Thus, this is also arisen by the secular perturbation from Planets.

The important difference between the effect of the self gravity and that of the secular perturbation from the Planets is that the self gravity can amplify the libration of resonant angle even though the particles initially have very small amplitude such as $A_{\sigma_{1: 2}}<30^{\circ}$. As Nesvorný and Roig (2001) have shown, the change of the amplitude of resonant angle which is initially $A_{\sigma_{1: 2}}<30^{\circ}$ practically unchanged by the secular perturbation of the planets for the age of Solar System. On the other hand, the amplitude increases by the self gravity even though particles initially have low amplitude. Figure 2 shows all particles initially satisfy the condition, $A_{\sigma_{1: 2}}<30^{\circ}$.

The time evolution of the fraction of the "stable" planetesimals in Model 1 is shown in the top of figure 6 . We refer the condition,

$$
A_{\sigma_{1: 2}}>30^{\circ}
$$

as "unstable condition" and "stable" planetesimals means the planetesimals which does not satisfy this con- 
dition. As Nesvorný and Roig (2001) insists, the chaotic evolution of the planetesimals which have the large amplitude of resonant angle becomes very strong and many of them are expected to escape from the 1:2 MMR. The condition, $A_{\sigma_{1: 2}}>30^{\circ}$ can be used as the crude threshold to determine whether planetesimal is unstable or not. The fraction of stable particles is calculated with respect to the particles which have $0.05<e$, $0.05<e<0.15,0.15<e<0.25$, and $0.25<e<0.35$. At first, each region roughly contains $920,140,320$, 460, particles, respectively and these numbers hardly change during the integration. For the planetesimals in the low eccentricity region $(0.05<e<0.15)$, The fraction of the stable particles quickly decreases. As the eccentricity increases the change of the fraction becomes slower. This comes from the difference of velocity dispersions. The bottom of the figure 6 shows the time evolution of the averaged amplitude of resonant angle. The average is taken over the particles at each eccentricity region. The resonant angle of particles in the low eccentricity region increases quicker than that of high eccentricity.

The solid line of the top panel shows that more than half of planetesimals in the 1:2 MMR satisfy the unstable condition within the $4.0 \times 10^{6}$ years in model 1 . This value can not be directly applied to real primordial TNOs because the parameter of our simulation is different from the real TNOs. Thus, how this result can extrapolate to realistic case must be discussed.

The top of the figure 7 shows how the fraction depends on the total mass of Twotinos. We vary the total mass as $2 M_{\text {tot }}$ (model 2), $M_{\text {tot }}$ (model 1), $0.5 M_{\text {tot }}$ (model 3) where $M_{t o t}$ is the total mass of the model 1 and its value is about $0.1 M_{\oplus}$. The particles which have $e>0.05$ are used to calculate the fraction. In the bottom of the figure 7, we plot the total mass vs the "half-life period" which is the time in which more than $50 \%$ of particles satisfy the unstable condition in logarithm scale. We fit the points with the linear curve $\log _{10} y=a \log _{10} x+b$ by least-squares method and obtain the value of the gradient, $a=-1.13152 \pm 0.1426(12.6 \%)$.

The figure 8 shows the dependency on the mass of the particles of the half-life period. The values of the mass is $2.0 M$ (model 5), 1.0M (model 1), $0.5 M$ (model 4), respectively. Where $M_{0}$ indicate the mass of each planetesimal of model 1 and its value is about $7.6 \times 10^{20} \mathrm{~kg}$. The bottom of the figure 8 shows the $\log (\operatorname{Mass})-$ $\log$ (halflife) relation. We fit the points same as above and the gradient is $a=-0.9693 \pm 0.0844(8.707 \%)$. These results are good agreement with the general feature of the relaxation timescale for the gravitational interaction, $t_{\text {relax }} \propto\left(m_{p} M_{t o t}\right)^{-1}$. Thus, we conclude the relaxation time of the amplitude of resonant angle also proportional to $\left(m_{p} M_{t o t}\right)^{-1}$.

The figure 9 shows how the timescale is changed by the inclination dispersion. The planetesimals whose eccentricity is higher than 0.05 are considered. The each line in the top panel shows the cases the dispersion, $\left\langle i^{2}\right\rangle^{1 / 2}$ are $0.002,0.01$ and 0.02 , respectively. We plot the inclination dispersion vs the time at which $25 \%$ of the particles satisfy the unstable conditions in logarithm scale in the bottom panel. the gradient, $a=$ $0.962302 \pm 0.03508(3.645 \%)$. With the above results, we conclude that the half-life period is proportional

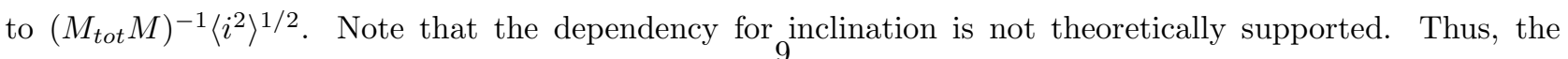


dependency for inclination is just phenomenalistic result and is not justified for all parameter space. But our extrapolation for inclination is up to one order of magnitude and our claims will be justified.

Our results can be summarized in the formula,

$$
t_{\text {half }}=4 \times 10^{6}\left(\frac{M_{\text {tot }}}{0.1 M_{\oplus}}\right)^{-1}\left(\frac{M_{p}}{7.6 \times 10^{23} g}\right)^{-1}\left(\frac{\left\langle i^{2}\right\rangle^{1 / 2}}{0.002}\right)(\text { years }) .
$$

We can estimate the unstable time-scale for realistic systems using this relation and the results we show above.

At first, we apply our result to the parameter of current Twotinos. Trujillo et al. (2001) estimate that the total mass of CTNOs is about $0.03 M_{\odot}$ and the population ratio of the classical TNOs and Twotinos are 1.0:0.07. They also pointed out that the differential size distribution of CTNOs is power law with exponent, $q \simeq 4.0$. This correspond to the power law differential mass distribution whose exponent is about 2.0. In this case, the main contribution of gravitational effect is come from the maximum size. If we assume the mass distribution is same between CTNOs and Twotinos, the total mass of Twotinos is about $0.002 M_{\oplus}$ and $m_{p}$ can be assumed to be maximum mass of Twotinos.

The maximum size of Twotinos is not well understood. We assume the maximum diameter of Twotinos is about $400 \mathrm{~km}$, this is equal to the estimated diameter of largest Twotinos, $2002 \mathrm{WC}_{19}$. The inclination dispersion of Twotinos assumed to be $5 \mathrm{deg}$. From eq 5 , we can estimate the half-life period of real Twotinos is about $3 \times 10^{11}$ years. This value is very large compared to the age of Solar system. Thus, we conclude that the self gravity effect is not important at the present day.

Next, we consider more drastic case in which the total mass of primordial Twotinos is about $3 M_{\oplus}$. This is the similar case Levison and Morbidelli (2003) assumed and the natural outcome if the planetesimals initially distribute up to $47 \mathrm{AU}$ with the power law density profile of minimum-mass solar nebular model (Hayashi 1981) and Neptune migrated very smoothly. We assume that the largest size of Twotinos is still about $400 \mathrm{~km}$. This is very modest assumption. The inclination is assumed to be order 1 deg again. This is also very modest assumption for bulk of primordial Twotinos because it is very rare to gain high inclination during migration (Gomes 2000). The power law exponent is assumed to be $q \simeq 4.0$. Then, the half-life period of this case become about $10^{6}$ year. This is sufficiently small compared to the age of Solar system. We can crudely estimate the rate of the particles which remain the resonance for the age of Solar system using this result. The half of particles in the resonance becomes unstable within the first $10^{6}$ year and the total mass of stable planetesimals roughly halves and the half-life period doubles, if we neglect the contribution which come into stable region. In the successive $2 \times 10^{6}$ years, the half of particles in the stable region becomes unstable, thus totally $75 \%$ of particles becomes unstable for $3 \times 10^{6}$ years. By iterating this process the mass of Twotinos can be reduce until the half-life period become comparable to $10^{9}$ years. This is significant result and invoke the reexamination to previous works which try to explain the dynamical structure of TNOs. Note that this kind of orbital instability never occur by the secular perturbation alone because the self gravity can amplify the libration of the resonant 
angle of the particles which initially have small amplitude (Nesvorný \& Roig 2001).

From above estimate, We conclude that the total mass of Twotinos decreases until the order of $0.01 M_{\oplus}$ even if Twotinos initially contain huge mass because the effect of self gravity is proportional to the total mass and the total mass decreases until the half-life period becomes comparable to the age of solar system. This estimate has large uncertainty because of the lack of sufficient survey of Twotinos.

If the index of the size distribution, $\mathrm{q}$ is proven to be larger than 4 for Twotinos, the largest objects do not contain the majority of the mass. In this case, the total mass must be adjusted down to include only the mass in those maximum mass planetesimals and the half-life period increases. Thus, the estimated value of timescale can change depending on the value of exponent, $q$ and the $M_{t o t}$ should be interpreted as total mass of large bodies. The value of $q$ is not well determined. For example, Petit et al. (2006) estimate the power law index for TNOs, $q \simeq 4.8$ and much larger than the result of Trujillo et al. (2002). Recently, theoretical work about the size distribution of TNOs have done and it suggests that $q \simeq 4.0$ (Sclichting \& Sari 2010). Further observational and theoretical work is required to determine the half-life period more exactly.

We point out the possibility that the high existing rate of high inclination population in 1:2 MMR is stem from the selective escape of the low inclination bodies. During orbital migration of Neptune, some of primordial Twotinos may gain high inclination (say, about $10 \mathrm{deg}$ ) with the similar effect proposed by Gomes (2000). This mechanism is very rare and it seems to be difficult to explain the large inclination of Twotinos with this mechanism alone. But if there was huge amount planetesimals between $30 \mathrm{AU}$ and $47 \mathrm{AU}$, the mechanism can provide sufficient number of planetesimals to account for the present high inclination population of Twotinos. After the migration, low inclination bodies selectively escape from 1:2 MMR because half-life period of low inclination body is an order of magnitude lower than high inclination body. Consequently, high inclination bodies selectively remain at 1:2 MMR. This can be a explanation why most of planetesimals of 1:2 MMR have high inclination.

\section{Summary and Discussion}

In this paper, we showed the results of the simulations which investigated the self gravity effect on Twotinos. We confirm the self gravity does change the resonant angle of planetesimals. We investigated how the timescale in which Twotinos becomes unstable depend on the total mass of Twotinos, the mass of planetesimals, and the inclination dispersion. The timescale obey the formula,

$$
t_{\text {half }}=4 \times 10^{6}\left(\frac{M_{\text {tot }}}{0.1 M_{\oplus}}\right)^{-1}\left(\frac{m_{p}}{7.6 \times 10^{23} g}\right)^{-1}\left(\frac{\left\langle i^{2}\right\rangle^{1 / 2}}{0.002}\right)(\text { years }),
$$

Here, $M_{t o t}$ is interpreted as the total mass at large sizes and $m_{p}$ is the mass of large bodies. $\left\langle i^{2}\right\rangle^{1 / 2}$ are the inclination dispersion.

Using this formula, we concluded that the total mass of Twotinos can be reduced to the order of $0.01 M_{\oplus}$ 

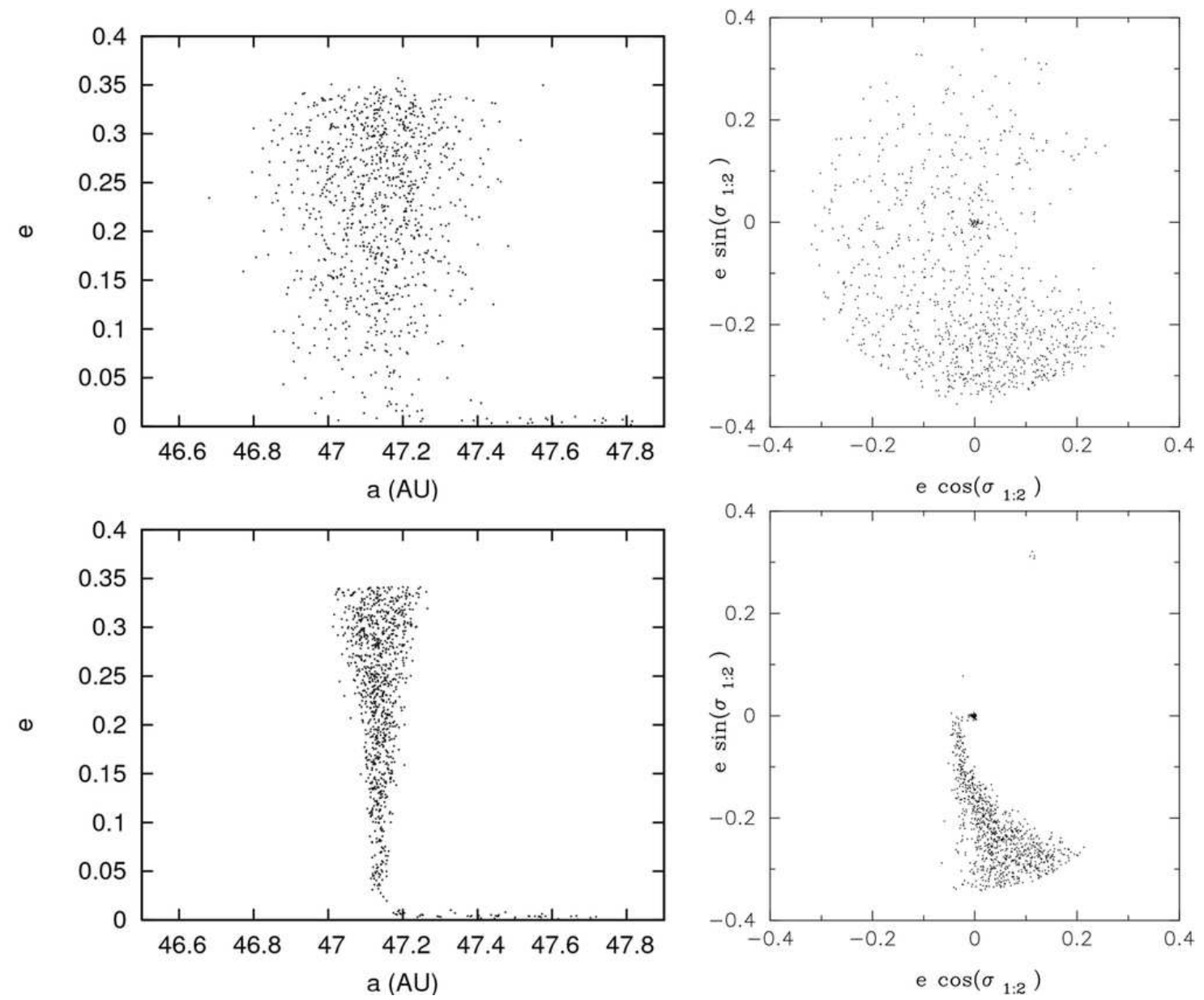

Figure 3: The planetesimals distribution at $t=1.6 \times 10^{7}$ years. The top left and the top right are plots on a-e plane and $e \cos \left(\sigma_{1: 2}\right)-e \sin \left(\sigma_{1: 2}\right)$ plane of model 1 , respectively. The bottom left and bottom right are the same plots of model 8 (the massless case). 

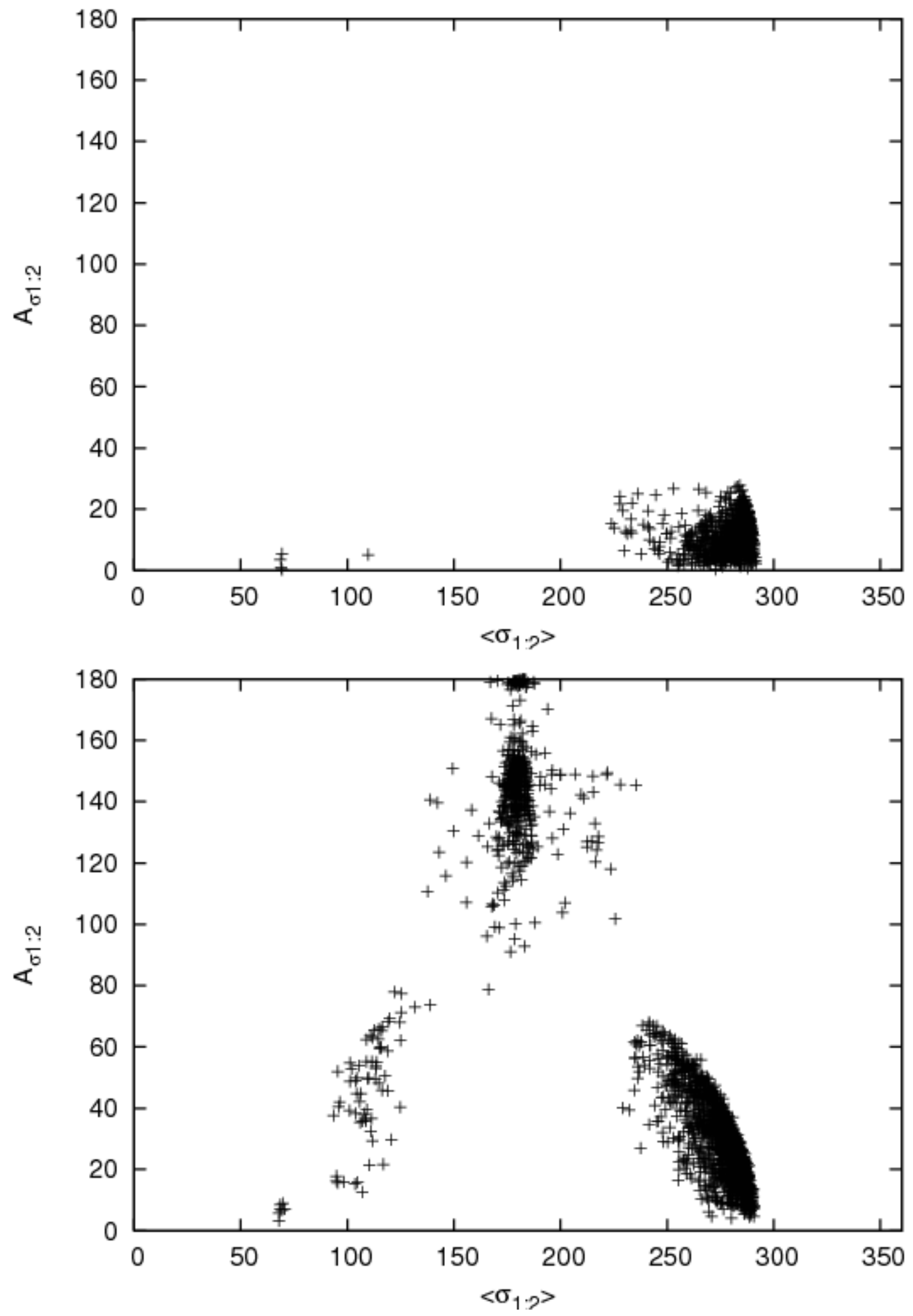

Figure 4: The planetesimals distribution on $\left\langle\sigma_{1: 2}\right\rangle-A_{\sigma_{1: 2}}$ plane at $t=1.6 \times 10^{7}$ years is shown. The top and bottom panel shows Model 8 and 1 respectively. the mean value, $\left\langle\sigma_{1: 2}\right\rangle$ is averaged over 160,000 years and the amplitude is defined as $\left(\sigma_{\max }-\sigma_{\min }\right) / 2$, where $\sigma_{\max }$ and $\sigma_{\min }$ are maximum and minimum value of resonant angle in each 160,000 year. 

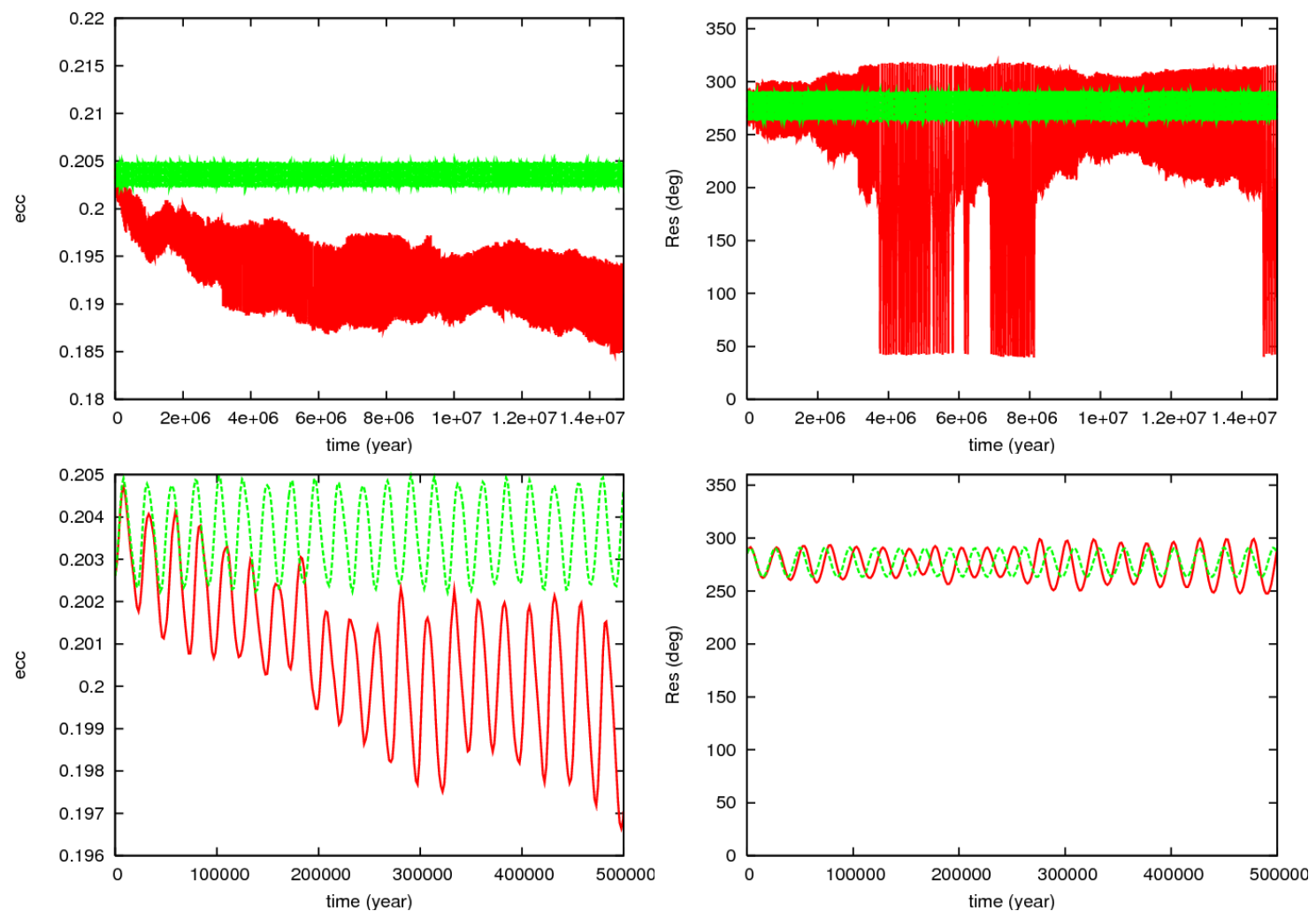

Figure 5: The time evolution of the resonant angle and eccentricity of a planetesimal. The top panels shows the long term evolution of the eccentricity (left) and the resonant angle (right). The time range of top panels is $\left[0: 1.5 \times 10^{7}\right]$ years. The bottom panels shows the short range evolution of the the eccentricity (left) and the resonant angle (right). The time range of the bottom panels is $\left[0: 5 \times 10^{5}\right]$ years 

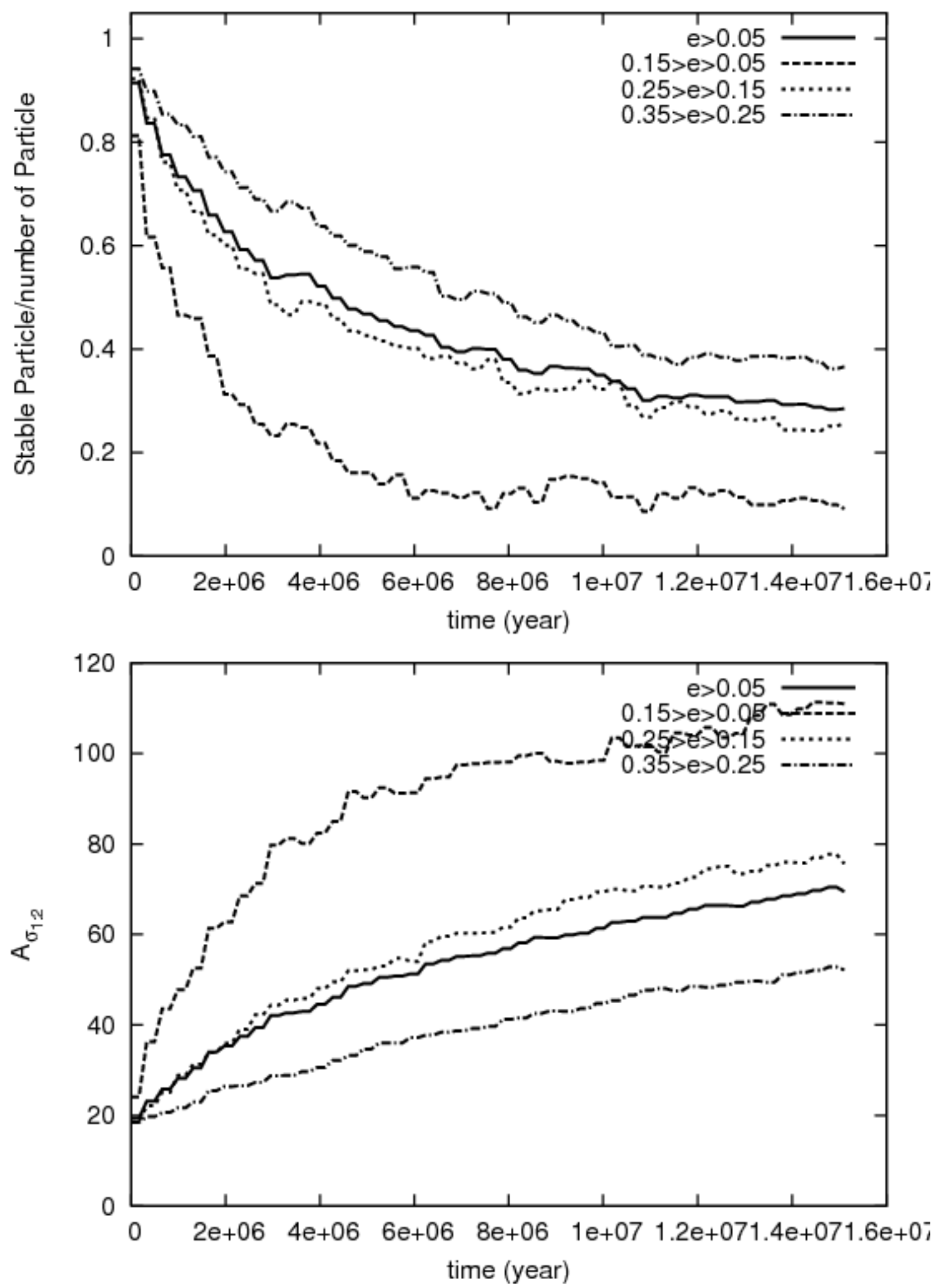

Figure 6: The time evolution of the fraction of the "stable" particles which does not satisfy the unstable condition (44)(top) and the averaged value of the amplitude of resonant angle (bottom). The average is taken with respect to the particles which have similar eccentricity. The solid, dashed, dotted, dot-dash line show the results of $0.05<e, 0.05<e<0.15,0.15<e<0.25$ and $0.25<e<0.35$, respectively. 

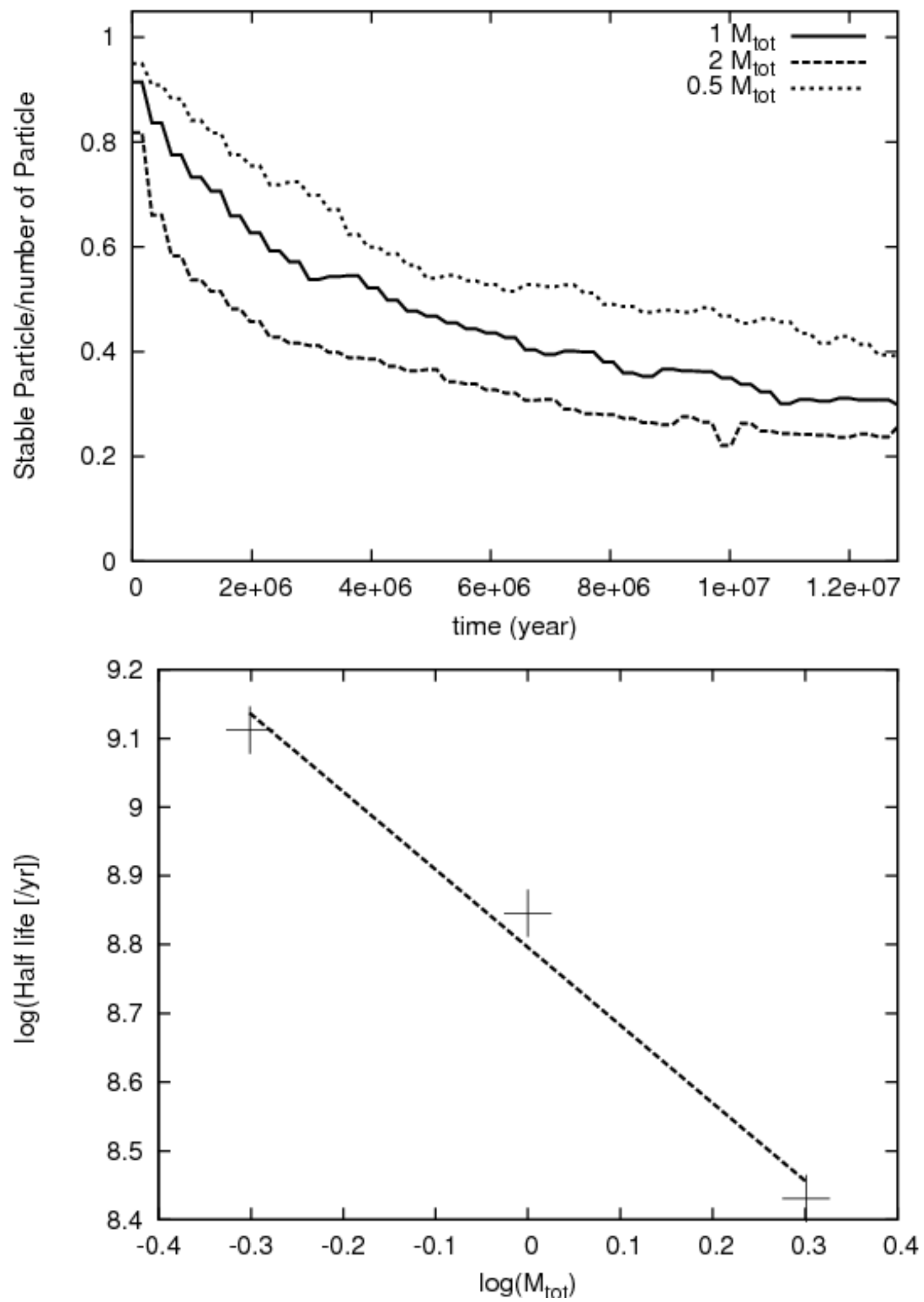

Figure 7: The top panel is the same as the top of figure 6 but the solid, dashed, dotted line are the result of model 1, model 4, model 5. The total mass of each model is $1 M_{t o t}$ (model 1 , solid), $2 M_{t o t}$ (model 4 , dashed), $0.5 M_{t o t}$ (model 5 , dotted), respectively and other parameters are the same. the results obtained using the particles whose eccentricity is $e>0.05$. $M_{t o t}$ is the total mass of model 1 and its value is about $0.1 M_{\oplus}$. The bottom panel shows the result of linear regression. We plot the total mass of Twotinos vs the "half-life period" in log scale. The gradient of the linear curve $a$ is $a=-1.13152 \pm 0.1426(12.6 \%)$ 

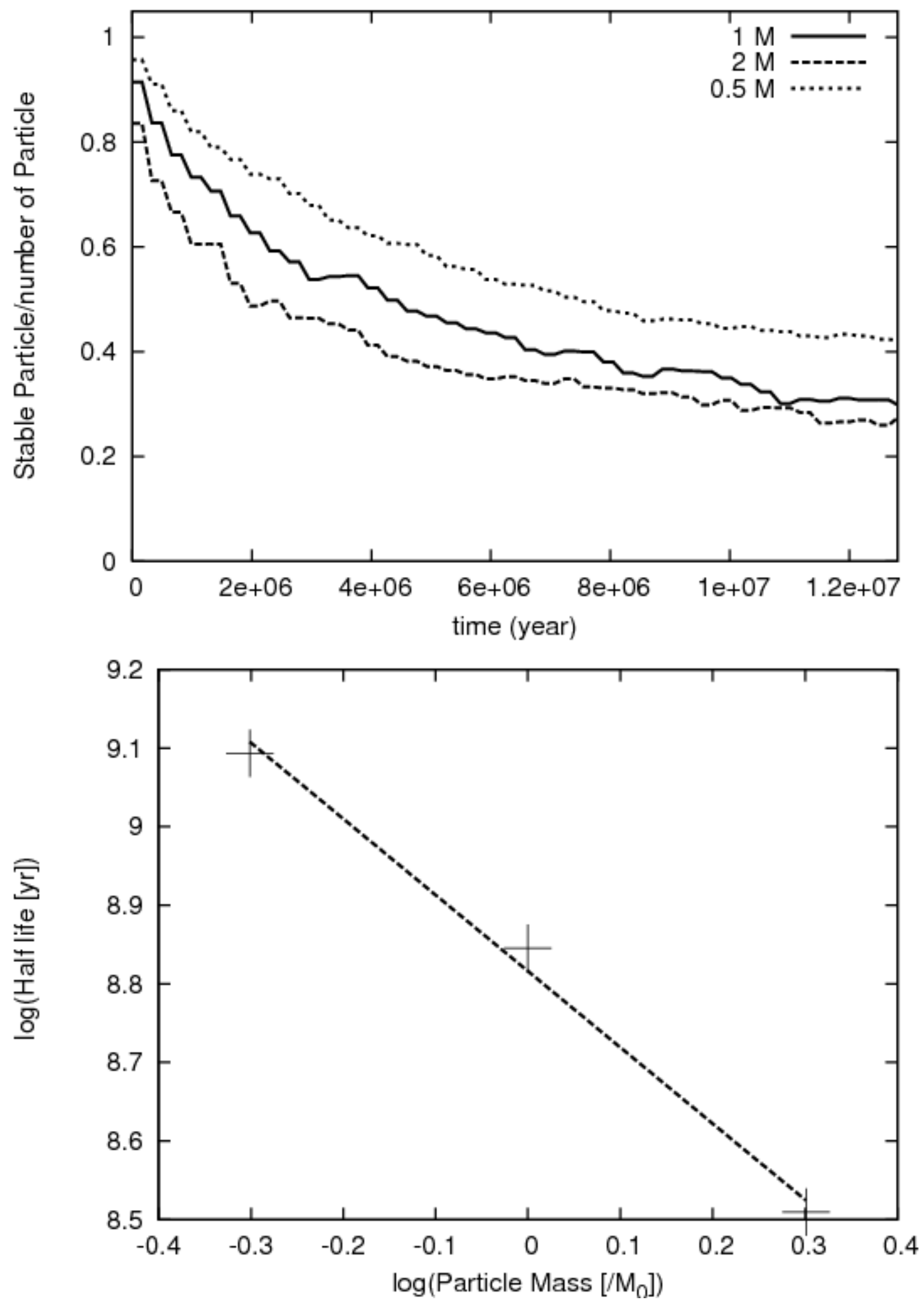

Figure 8: The top panel is the same as the top of figure 6] but the solid, dashed, dotted line are the results of model 1, 2, 3, respectively. The mass of planetesimals of each model is $M_{0}, 2 M_{0}, 0.5 M_{0}$, respectively and other parameters are the same. The results obtained using the particles whose eccentricity is $e>0.05$. The $M_{0}$ is the mass of particle of model 1 and its value is about $7.62 \times 10^{23} \mathrm{~g}$. The bottom panel shows the result of linear regression. We plot the mass of each planetesimal vs the "half-life period" in log scale. The gradient of the linear curve $a$ is $a=-0.9693 \pm 0.0844(8.707 \%)$. 

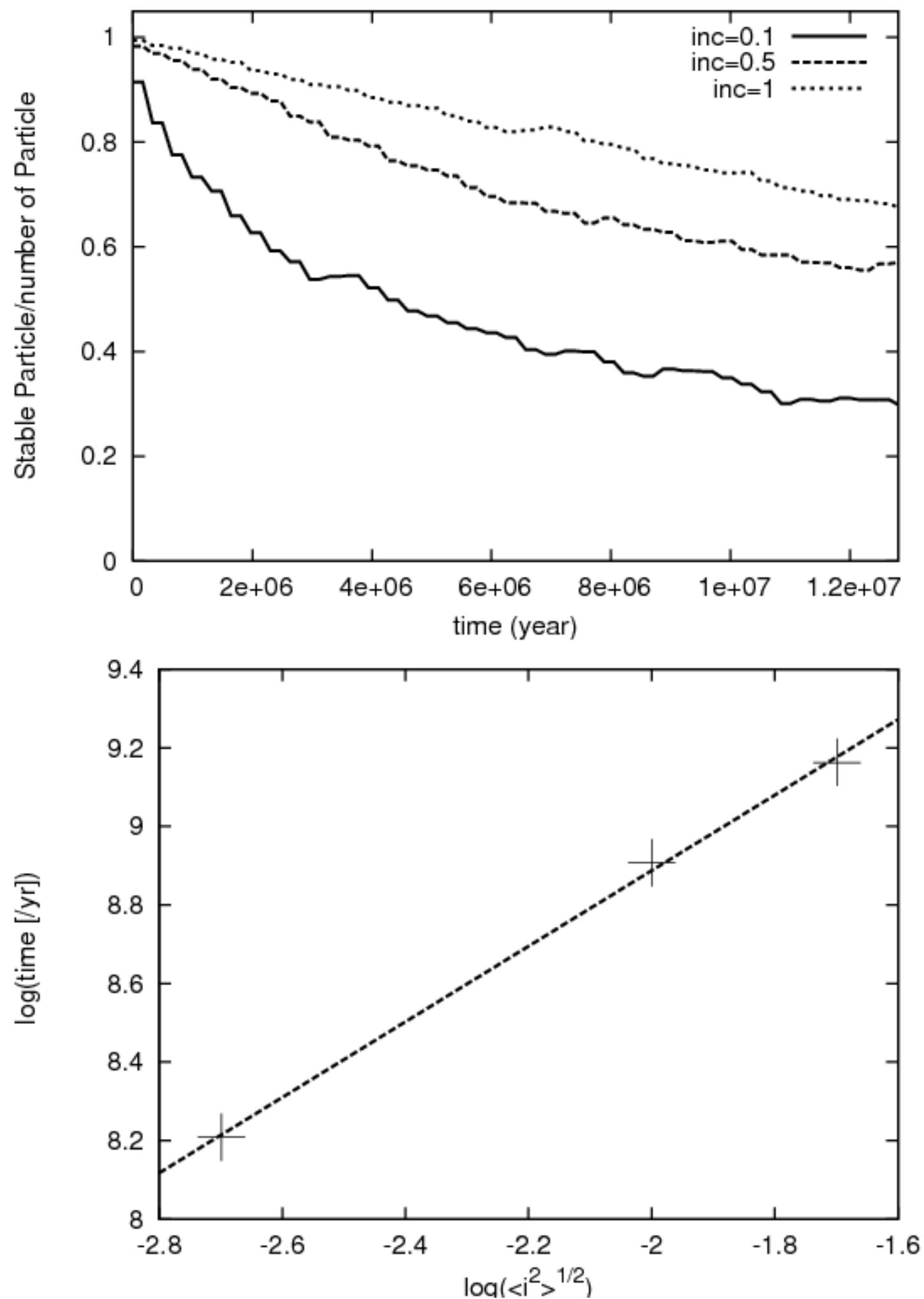

Figure 9: The top panel is the same as the top of figure 6] but the solid, dashed, dotted line are the results of model 1,6 , 7 . The inclination dispersion, $\left\langle i^{2}\right\rangle^{1 / 2}$ of each model is $0.002,0.01,0.02$, respectively and other parameters are the same. The results obtained using the particles whose eccentricity is $e>0.05$. The bottom panel shows the result of linear regression. We plot the inclination dispersion vs the time in which the $25 \%$ of particles become unstable in log scale. The gradient of the linear curve $a$ is $a=0.962302 \pm 0.03508(3.645 \%)$. 
even if there was huge mass such as several order of earth mass in the 1:2 MMR because the effect of self gravity is proportional to the total mass and sufficiently strong until the total mass fall bellow the threshold mass at which the timescale becomes comparable to the age of solar system. These results invoke reexamination to many previous works such as the dynamical evolution model of TNOs (Levison and Morbidelli 2003) or Neptune migration speed estimated from the current orbital population of Twotinos (Murray-Clay \& Chiang 2005).

The work of Levison \& Morbidelli (2003) relies on the mass carrying effect of the 1:2 MMR to explain the structure of the TNOs region. With the results done by Gomes (2003), their result successfully explain the important properties of TNOs. The model assumes that the total mass of 1:2 MMR population is about $3 M_{\oplus}$ when Neptune is at $22 \mathrm{AU}$ and secular resonance between Twotinos and Neptune induce the oscillation of eccentricity of Twotinos. When the planetesimals reach small eccentricity, Neptune's stochastic migration kick out these bodies from resonance. This is the origin of the cold population in CTNOs. They also argue that almost 1:2 MMR population also kicked out during the migration process by the stochastic migration. But this argument contains self-contradiction. As many works have shown (Hahn \& Malhotra 1999, Zhou et al. 2002), if Neptune's migration was much jumpy to shake off the bulk of the planetesimals in the 1:2 MMR, it seems to impossible to capture the planetesimals into the 1:2 MMR. Further more, Murray-Clay \& Chiang (2006) pointed out that primordial planetesimal disk of TNOs region did not have big objects which can induce jumpy migration. This seems to be a serious problem of this model.

On the other hand, our results naturally explain the removing process from 1:2 MMR after Neptune migration finished. Further more the particles which has low eccentricity becomes unstable quicker than high eccentricity particles as we have shown. It can be an alternative mechanism to kick out the planetesimals into classical TNOs region. Thus it is unnecessary to assume the strongly junky migration if the self gravity effectively work and the model suggested by Levison \& Morbidelli (2003) becomes more attractive. Of course further research is needed to determine how the self gravity acts during the Neptune migration.

If the huge mass such as several order of earth mass is really captured and released from Twotinos, these particles may affects the additional migration to Neptune or other planets as Morbidelli et al. (2007) pointed out. This effects can change the entire structure of trans neptunian region and the scenario of the formation of our Solar system. To investigate the "second migration process" effects and how it will change the entire structure of trans Neptune region is beyond our scope in this paper and further investigations are required.

Murray-Clay \& Chiang (2005) estimate the migration speed of Neptune from the current population of 1:2 MMR. Their result show whether the planetesimals distribute on the "trailing island" or "leading island" whose liberation center of oscillation of resonant angle is greater than $\pi$ as opposed to less than $\pi$ sensitively depends on the timescale of Neptune migration. However, our results show that the planetesimals can "penetrate" from these island due to the self gravity and destroy the information of the primordial dynamical structure of Twotinos. Thus, we think more precise treatment is required to estimate the timescale of Neptune migrations 
from the population of Twotinos.

Our result is very strong because there is no arbitrary assumption and is natural outcome of physical principle. More investigations are required whether our results can be the key mechanism to explain the entire structure of trans neptunian region but we believe our result play very important role for the orbital evolution of TNOs.

\section{Acknowledgments}

We thank Masaki Iwasawa, Eichiro Kokubo and Junichiro Makino for fruitful discussions This research is partially supported by the Special Coordination Fund for Promoting Science and Technology (GRAPE-DR project). Ministry of Education, Culture, Sports, Science and Technology, Japan.

\section{References}

[1] Aarseth, S. J. 1985, in Multiple Time Scales, ed. J. U. Brackhill and B. I. Cohen (Academic Press, New York), p. 377.

[2] Chiang, E. I. and Jordan, A. B. (2002). On the Plutinos and Twotinos of the Kuiper Belt. AJ, 124:34303444 .

[3] GRAPE-6A: A Single-Card GRAPE-6 for Parallel PC-GRAPE Cluster Systems Fukushige, T., Makino, J., \& Kawai, A. 2005, PASJ, 57, 1009

[4] Gomes, R. S. 2000. Planetary migration and Plutino orbital inclinations Icarus 161, 404-418.

[5] Gomes, R. S. 2003. The origin of the Kuiper Belt high-inclination population. Icarus 161, 404-418.

[6] Gomes, R. S., Morbidelli, A., \& Levison, H. F. 2004, Planetary migration in a planetesimal disk: why did Neptune stop at 30 AU? Icarus, 170, 492

[7] Gomes, R., Levison, H. F., Tsiganis, K., \& Morbidelli, A. 2005, Origin of the cataclysmic Late Heavy Bombardment period of the terrestrial planets Nature, 435, 466

[8] Hahn, J. M., \& Malhotra, R. 1999, Orbital Evolution of Planets Embedded in a Planetesimal Disk AJ, 117,3041

[9] Ida, S., Bryden, G., Lin, D. N. C., \& Tanaka, H. 2000, Orbital Migration of Neptune and orbital distribution of trans-Neptunian objects ApJ, 534, 428

[10] Kokubo, E., Yoshinaga, K., \& Makino, J. 1998, On a time-symmetric Hermite integrator for planetary N-body simulation MNRAS,297, 1067 
[11] Levison, H. F., Morbidelli, A. 2003, The formation of the Kuiper belt by the outward transport of bodies during Neptune's migration Nature, 426, 419

[12] Levison, H. F.; Stern, S. A. 1995 Possible origin and early dynamical evolution of the Pluto-Charon Binary. Icarus, 116, 315

[13] Makino, J. 1991, A Modified Aarseth Code for GRAPE and Vector Processors PASJ, 43, 859

[14] Makino, J., \& Aarseth, S. J. 1992, On a Hermite integrator with Ahmad-Cohen scheme for gravitational many-body problems PASJ, 44, 141

[15] Makino, J., Fukushige, T., Koga, M., \& Namura, K. 2003, GRAPE-6: Massively-Parallel Special-Purpose Computer for Astrophysical Particle Simulations PASJ, 55, 1163

[16] Malhotra, R. 1995. The origin of Pluto's orbit: Implications for the solar system beyond Neptune. AJ, 110:420.

[17] Minton, D. A.; Malhotra, R 2009, A record of planet migration in the main asteroid belt Natur, 457 ,1109

[18] Morbidelli, A. (1997). Chaotic diffusion and the origin of comets from the $2 / 3$ resonance in the Kuiper Belt. Icarus, 127:1-12.

[19] Morbidelli, A., Levison, H. F., Tsiganis, K., Gomes, R. 2005. Chaotic capture of Jupiter's Trojan asteroids in the early Solar System. Nature 435, 462-465.

[20] Murray-Clay, R. A., \& Chiang, E. I. Brownian motion in Planetary migration 2006, ApJ, 651, 1194

[21] Murray-Clay, R. A., \& Chiang, E. I. A Signature of Planetary Migration: The Origin of Asymmetric Capture in the 2:1 Resonance 2005, ApJ, 619, 623

[22] Nesvorný, D. and Roig, F. (2000). Mean motion resonances in the trans-Neptunian region I. The 2:3 resonance with Neptune. Icarus, 148:282-300.

[23] Nesvorný, D. and Roig, F. (2001). Mean motion resonances in the trans-Neptunian region II. The 1:2, 3:4, and weaker resonances. Icarus, 150:104-123.

[24] Petit, J.-M.; Holman, M. J.; Gladman, B. J.; Kavelaars, J. J.; Scholl, H.; Loredo, T. J. The Kuiper Belt luminosity function from $\mathrm{mR}=22$ to 252006 , MNRAS, 365, 429

[25] Schlichting, H. E., Sari, R. 2010 Runaway Growth Dring Planet Formation: Explaining the Size Distribution of Large Kuiper Belt Objects 2010arXiv1011.0201S

[26] Tiscareno, M. S., Marhotra, R, 2009 Chaotic Diffusion of Resonant Kuiper Belt Objects AJ, 138,827 
[27] Trujillo, C. A., Brown, M. E., Bouchez, A. H., 2001, Large Bodies in the Kuiper Belt AJ, 122, 2740

[28] Tsiganis, K., Gomes, R., Morbidelli, A., \& Levison, H. F. 2005, Origin of the orbital architecture of the giant planets of the Solar System Nature, 435, 459

[29] Zhou, L.-Y., Sun, Y.-S. Zhou, J.-L., Zheng, J.Q., \& Valtonen, M. 2002, Stochastic effects in the planet migration and orbital distribution of the Kuiper Belt MNRAS, 336, 520 